\title{
Elevated pentose phosphate pathway is involved in the recovery of hypoxia-induced erythrocytosis
}

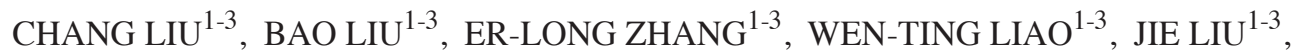 \\ BING-DA SUN ${ }^{1-3}$, GANG XU ${ }^{1-3}$, JIAN CHEN ${ }^{1-3}$ and YU-QI GAO ${ }^{1-3}$ \\ ${ }^{1}$ Institute of Medicine and Hygienic Equipment for High Altitude Region, College of High Altitude Military Medicine, \\ Third Military Medical University; ${ }^{2}$ Key Laboratory of High Altitude Environmental Medicine; \\ ${ }^{3}$ Key Laboratory of High Altitude Medicine, PLA, Chongqing 400038, P.R. China
}

Received March 15, 2017; Accepted September 27, 2017

DOI: $10.3892 / \mathrm{mmr} .2017 .7801$

\begin{abstract}
As a typical model of hypoxia-induced excessive erythrocytosis, high altitude polycythemia (HAPC) results in microcirculation disturbance, aggravates tissue hypoxia and results in a severe clinical outcome, without any effective intervention methods except for returning to an oxygen-rich environment. The present study aimed to explore potential therapeutic targets which may participate in the recovery of HAPC by studying the mechanisms of reducing the hemoglobin (HB) concentration during re-oxygenation. A total of 14 and 13 subjects were recruited over a 5,300 $\mathrm{m}$ distance and 5,170 $\mathrm{m}$ area. The patients were classified into HAPC or control groups based on their HB value. Plasma samples were collected on the day when they finished their stay in plateau for a year, and on the 180th day following their reaching in plain. Metabolic profiling was conducted by UPLC-QTOF/MS. MetaboAnalyst platform was performed to explore the most perturbed metabolic pathways. A panel of differential metabolites were obtained in the recovery phase of HAPC and control groups. The present study identified the uniquely upregulated pentose phosphate pathway in HAPC subjects, along with a significantly decreased HB level. The findings were verified via a direct comparison between HAPC and control subjects at a high altitude. An increased pentose phosphate pathway was identified in control groups compared with HAPC subjects. An elevated pentose phosphate pathway may therefore participate in the recovery of HAPC, whereas a downregulated pentose phosphate pathway may contribute to hypoxia-induced erythrocytosis. The results of the present study provide potential therapeutic strategies and novel insights into the pathogenesis of hypoxia-induced polycythemia.
\end{abstract}

Correspondence to: Mr. Jian Chen or Yu-Qi Gao, Institute of Medicine and Hygienic Equipment for High Altitude Region, College of High Altitude Military Medicine, Third Military Medical University, 30 Gao Tanyan Street, Chongqing 400038, P.R. China

E-mail: jchenone@163.com

E-mail: gaoy66@yahoo.com

Key words: serum, metabolomics, hypoxia, polycythemia, recovery, UPLC-QTOF/MS

\section{Introduction}

Excessive erythropoiesis refers to an increase of mature erythrocytes in peripheral circulation. Overburdened hemoglobin (HB) increases blood viscosity, microcirculation disturbance and worsens organ hypoxia, augmenting clinical risks of multiple ischemic diseases including stroke, thrombosis (1). Excessive erythropoiesis could be classified into idiopathic or acquired pathological symptoms for different pathogenesis. Specifically, hypoxia has been considered as a major stimulating factor for secondary polycythemia. It is generally accepted that, in hypoxic environment, hypoxia-inducible factor is upregulated and serves as an oxygen-dependent regulator which stimulates erythropoietin production in hematopoietic organs, enhances iron uptake/utilization, and facilitates maturation and proliferation of erythroid progenitor, further lead to erythropoiesis $(2,3)$.

The syndrome with high $\mathrm{HB}$ (males, $\mathrm{HB} \geq 21 \mathrm{~g} / \mathrm{dl}$; females, $\mathrm{HB} \geq 19 \mathrm{~g} / \mathrm{dl}$ ) in long living plateau residents, is called high altitude polycythemia (HAPC) (4). As one of the typical models of secondary erythrocytosis, HAPC model is widely employed in scientific researches of hypoxia-induced excessive erythropoiesis, with the performance of effective generalization and high precision (5). In addition, epidemiological study has shown that HAPC subjects accounted for nearly $10 \%$ of the population residing in Tibet (6). Although HAPC poses a great threat to these individuals' life security and quality, no effective therapeutic methods could be applied for these subjects except for long term oxygen therapy or returning to plain (7). Therefore, it is urgently needed to explore the mechanisms and potential therapeutic biomarkers in relieving HAPC.

Metabolomics, as a promising approach in systemic biology, offers a systematic description of low-molecular weight molecules from samples and is typically used to monitor the response of cells or organism to external stimuli (8). Metabolomics provides valuable methods of scientific investigations in exploring global alterations enrolled in the occurrence and development of diseases. Importantly, derived from metabolic reactions of individuals themselves, distinguishing molecules detected in metabolomics pose greater clinical significances (9). To date, metabolomics has 
exhibited enormous roles in exploring hypoxic diseases while only one study focused on polycythemia which suggested that familial VHL ${ }^{\mathrm{R} 200 \mathrm{~W}}$ homozygotes with elevated citrate and glycerol showed a greater risk in suffering erythrocytes hyperplasia (10). A comprehensive understanding in metabolomics profiling of HAPC subjects is urgently needed.

In this pilot study, the objective of our research is to search for distinguishing molecules and related pathways in the recovery phase of HAPC subjects. Hence, we conducted a non-targeted UPLC-QTOF/MS method on serum samples from HAPC and control individuals and described dynamic alterations of these metabolites during re-oxygenation. To eliminate the influence of environment and living habits, we introduced heterogeneous samples from two different locations and employed Venn figures to search for distinguishing molecules of HAPC. Additionally, we attempted to explore if these potential biomarkers are involved in the progression of excessive erythropoiesis. Our findings may contribute to a better understanding of mechanisms and potential therapeutic strategies for hypoxia-induced excessive erythrocytosis.

\section{Materials and methods}

Subjects and experimental procedures. A total of twenty-seven subjects were recruited in this study, including 14 subjects from the location of $5300 \mathrm{~m}$ (represented by letter $\mathrm{K}$ ) and 13 residents from the area of $5170 \mathrm{~m}$ (represented by letter $\mathrm{T}$ ), respectively, which share a close range of latitude, identical geographical and climatic environment. The inclusion and exclusion criteria were as follows: i) age $\geq 18$ years; ii) at least 12 -month experience in their respective residences; iii) participants from plains; iv) none exposure history to high altitude before; v) none medical intervention for excessive erythropoiesis during recovery period; vi) controls were defined as subjects with $\mathrm{HB}$ level $<21 \mathrm{~g} / \mathrm{dl}$; vii) individuals with $\mathrm{HB}$ reaching $21 \mathrm{~g} / \mathrm{dl}$ were included in experimental group. None statistical differences could be observed for age, oxygen saturation, oxygen saturation, heart rate, diastolic blood pressure, and systolic blood pressure, among subjects from these two locations. Written informed consent was obtained from all volunteers. The present study received ethical approval from the medical ethical committee of the Third Military Medical University. The entire experiment was complied with the principles of the Declaration of Helsinki.

Plasma sample preparation. Enrolled participants shared identical diet and activity arrangements during the whole experiment in these separating locations. Plasmas samples were obtained at the beginning of this trial on the 1st day assigned and 180th day after their arriving at the plain. Sampling strategies and preparation methods are identical to our previous report (11). Briefly, morning fasting venous blood $(100 \mu \mathrm{l})$ was collected with EDTA as an anticoagulant, and centrifuged to separate serum at $14,000 \mathrm{x}$ g for $15 \mathrm{~min}$ at $4^{\circ} \mathrm{C}$. After the process of fast frozen, these plasma samples were delivered to Chongqing for further metabolomics analysis in the courier filled with dry ice. Processing procedure in this study was totally in accordance to the manufacturers' protocols of all devices.

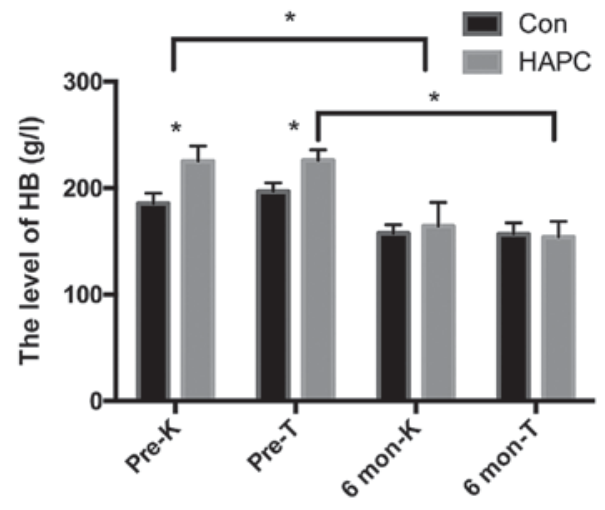

Figure 1. Characteristics of HB level. There exist significant differences in locations of $\mathrm{K}$ and $\mathrm{T}$ in value of $\mathrm{HB}$, respectively. HB level varied significantly before and after oxygenation in these two separate locations. ("P-value $<0.05$; P-value was calculated by student's t-test). HB, hemoglobin; HAPC, high altitude polycythemia; Con, control.
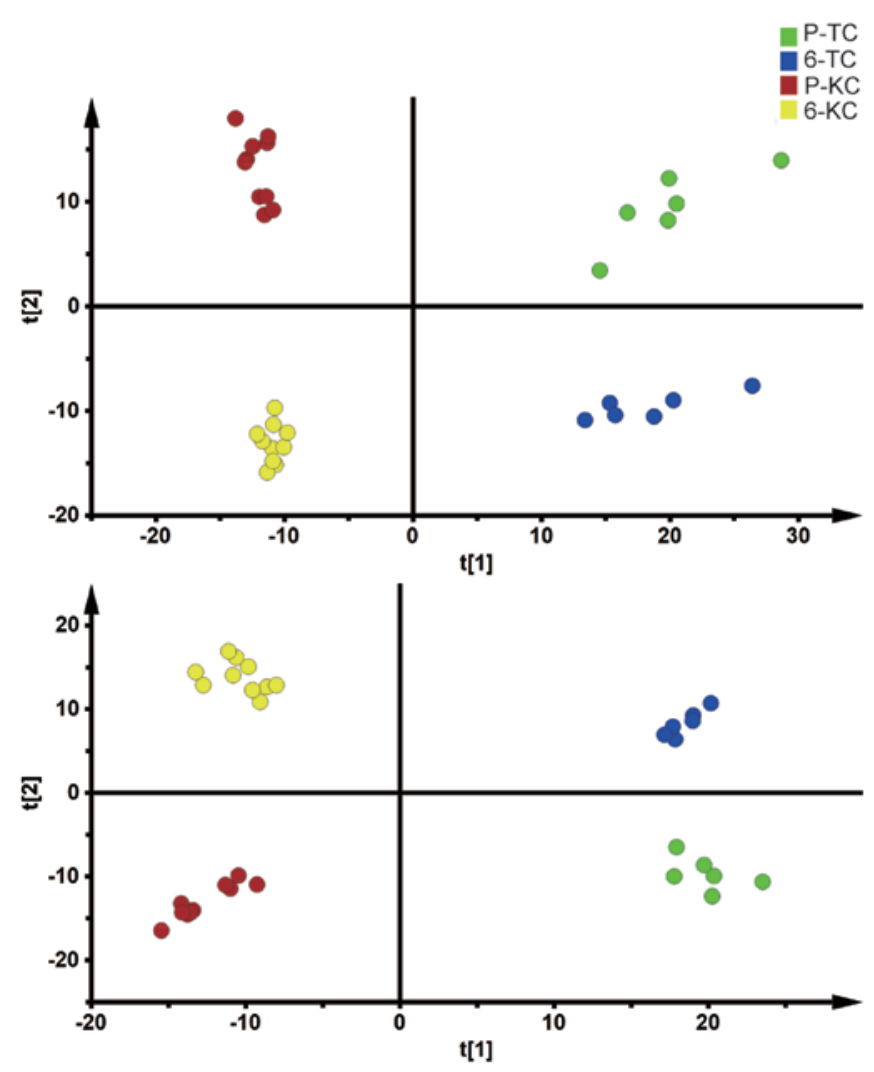

Figure 2. PCA and OPLSDA figures of subjects in Control groups. $\mathrm{P}$, pre-oxygenation; $\mathrm{K}$, from location $\mathrm{K}$; T, from point $\mathrm{T}$; C, control groups; H, HAPC group; OPLS-DA, partial squares discriminant analysis; PCA, principal component analysis; HAPC, high altitude polycythemia.

Metabolome analysis by UPLC-QTOFMS. The experimental procedures of UPLC-QTOFMS were identical to our published report with minor modifications (11). Briefly, metabolomics analysis was conducted on an Agilent 1290 Infinity LC system (Agilent, Santa Clara, CA, USA). Chromatographic separations were performed on an ACQUITY UHPLC HSS T3 C18 column ( $2.1 \times 100 \mathrm{~mm}, 1.8 \mu \mathrm{m}$; Waters, Milford, Ireland) at $45^{\circ} \mathrm{C}$. The electrospray ionization source interface was also performed in the optimized conditions after the exploration in 
Table I. Summary of parameters for OPLS-DA and PCA models.

\begin{tabular}{lrccc}
\hline Model name & $\mathrm{N}$ & $\mathrm{R} 2 \mathrm{X}$ & $\mathrm{R} 2 \mathrm{Y}$ & $\mathrm{Q} 2$ \\
\hline OPLSDA & & & & \\
KC-pos & 20 & 0.578 & 0.99 & 0.974 \\
KC-neg & 20 & 0.622 & 0.991 & 0.98 \\
TC-pos & 12 & 0.687 & 0.983 & 0.959 \\
TC-neg & 12 & 0.516 & 0.994 & 0.972 \\
KH-pos & 8 & 0.611 & 0.997 & 0.968 \\
KH-neg & 8 & 0.782 & 0.998 & 0.978 \\
TH-pos & 14 & 0.559 & 0.992 & 0.976 \\
TH-neg & 14 & 0.651 & 0.995 & 0.986 \\
PCA & & & & \\
KC-pos & 20 & 0.688 & & 0.473 \\
KC-neg & 20 & 0.625 & & 0.498 \\
TC-pos & 12 & 0.71 & & 0.484 \\
TC-neg & 12 & 0.683 & & 0.49 \\
KH-pos & 8 & 0.759 & & 0.242 \\
KH-neg & 8 & 0.702 & & 0.427 \\
TH-pos & 14 & 0.627 & & 0.419 \\
\hline
\end{tabular}

OPLS-DA, partial squares discriminant analysis; PCA, principal component analysis.

our prior studies, and to monitor as many ions as possible in both positive and negative modes.

Metabolomics data preprocessing. Data preprocessing and metabolomics analysis followed our previously published paper with minor modifications (11). Raw LC-MS data were converted to mzData formats via Agilent MassHunter Qualitative software. The program XCMS (version 1.40.0) was used to preprocess the raw data, including peak detection, peak matching, matched filtration, and nonlinear alignment of data, with the default parameters (fwhm, 10; bw, 10; and snthresh, 5). The internal standards were removed after the employment of these molecules in data quality control (reproducibility) and data normalization. The variables that did not present in at least $80 \%$ of groups were filtered. The resulting matrix consisting of retention time, mass-to-charge ratio $(\mathrm{m} / \mathrm{z})$ and normalized ion intensities, were introduced to the subsequent analysis.

Data processing and analysis. The preprocessed data matrix was exported to Matlab (MathWorks, Natick, MA) and SIMCA-P 13.0 (Umetrics Inc., Kinnelon, NJ) software for further analysis. Partial squares discriminant analysis (OPLS-DA) and principal component analysis (PCA) were carried out to visualize separation of preand post-re-oxygenation in metabolic profiling. Variable importance project (VIP) revealing discriminatory metabolites which enrolled in the classification effects of re-oxygenation intervention was calculated in this study. In addition, a paired t-test and fold-change value (FC) were executed to discovery metabolic features. To improve the statistical robustness, Bonferroni correction for multiple testing with an adjusted
P-value were also performed. The screening criteria of VIP $>1, q<0.05$ and fold-change value increased or decreased metabolites that changed by 1.5 -fold were considered as most responsible molecules, which were applied for further analysis in their potential implications in the HAPC. Experimental values in this study were carried out as mean \pm SD. Generally, P-value less than 0.05 was regarded as significant statistically, unless noted otherwise.

Metabolites identification and metabolic pathway analysis. Accurate mass measurements were subjected to database searching in the public databases METLIN (12) and HMDB (13), and subsequently matched with commercially available standard metabolites. Pathway analysis containing enrichment analysis and pathway topological analysis was established based on MetaboAnalyst platform (http://www.metaboanalyst.ca) (14).

\section{Results}

Characteristics of enrolled participants. HB levels from clinical routine blood test divided participants into four groups that HAPC group in location $\mathrm{K}$ contains 4 participants and HAPC group of location T contains 7 subjects, while others with HB levels below $21 \mathrm{~g} / \mathrm{dl}$ were enrolled in control groups of separating locations. Their recover conditions after reaching plain for 180 day were also measured by blood routine examination. Detailed description of HB in HAPC and control groups from these two separate places are presented in Fig. 1. HB levels differed markedly in HAPC and control groups of different locations, namely HAPC of location K, HAPC of location T, control group of location $\mathrm{K}$, control group of location $\mathrm{T}$, before and after re-oxygenation, separately. All recruitments showed ideal recovery status with significant decreasing $\mathrm{HB}$ in plain environment (P-value <0.05).

Serum metabolomics alterations in HAPC groups. For global metabolomic profiling in HAPC group, multivariate statistical analysis was performed to classify metabolic phenotypes and identify differentiating metabolites. As shown in the Fig. 2, a clear separation was achieved on PCA and OPLSDA scores plot based on spectral data in HAPC subjects in separating locations before and after restoring $\mathrm{HB}$ value to significantly lower level. Detailed information about multivariate model is listed in Table I.

Based on previous OPLS-DA models, variable importance in project (VIP) was extracted to facilitate the classification of re-oxygenation intervention. A series of methods including paired t-test, FDR and FC, were also performed to validate the significance of these discriminated variables.

Physiological alterations after re-oxygenation in metabolic profiles. To exclude influence of re-oxygenation on physiological alterations and gain a better understanding of mechanisms after re-oxygenation, the metabolic profiling in control groups from two locations were assessed separately. Fig. 3 illustrated the PCA and OPLSDA score plots for subjects in control groups based on spectral data of UPLC-QTOFMS. Detailed information about multivariate model is also listed in Table I. All these score plots showed clearly separation before and after the exposure to re-oxygenation. 
Table II. The uniquely significant metabolites of HAPC subjects in the recovery phases.

\begin{tabular}{lcccccccc}
\hline Molecules & P-value & Q-value & FC & VIP & P-value & Q-value & FC & VIP \\
\hline ESI+ & & & & & & & & \\
$\quad$ & & & & & & \\
Phosphoribosyl pyrophosphate & 0.0065 & 0.0039 & 2.0029 & 1.2778 & 0.0002 & 0.00010 & 1.9605 & 1.2735 \\
L-Tryptophan & 0.0006 & 0.0014 & 0.3693 & 1.3128 & $2.50 \mathrm{E}-05$ & $2.30 \mathrm{E}-0$ & 0.3586 & 1.4052 \\
Glucose 6-phosphate & 0.0051 & 0.0032 & 2.5161 & 1.2682 & $2.22 \mathrm{E}-06$ & $4.40 \mathrm{E}-0$ & 2.4762 & 1.3070 \\
3,4-Dihydroxyphenylglycol O-sulfate & 0.0073 & 0.0041 & 2.0014 & 1.2983 & $3.85 \mathrm{E}-05$ & $2.99 \mathrm{E}-0$ & 1.7201 & 1.3754 \\
LysoPE(0:0/22:2) & 0.0171 & 0.0072 & 2.0335 & 1.2243 & $6.25 \mathrm{E}-06$ & $8.83 \mathrm{E}-0$ & 1.6479 & 1.3319 \\
ESI- & & & & & & & & \\
Uracil & 0.0031 & 0.0024 & 1.5045 & 1.2163 & $2.24 \mathrm{E}-05$ & $2.16 \mathrm{E}-0$ & 4.2566 & 1.4169 \\
Deoxyribose 5-phosphate & 0.0028 & 0.0024 & 0.2604 & 1.2912 & $4.18 \mathrm{E}-05$ & $3.22 \mathrm{E}-0$ & 0.5764 & 1.3991 \\
All-trans-retinoic acid & 0.0037 & 0.0027 & 0.0173 & 1.3286 & $3.51 \mathrm{E}-05$ & $2.80 \mathrm{E}-0$ & 0.3724 & 1.3148 \\
LysoPE (0:0/22:6) & 0.0005 & 0.0014 & 0.4857 & 1.3031 & 0.001220 & 0.00047 & 0.5858 & 1.2854 \\
\hline
\end{tabular}

HAPC, high altitude polycythemia; FC, fold-change; VIP, variable importance project.
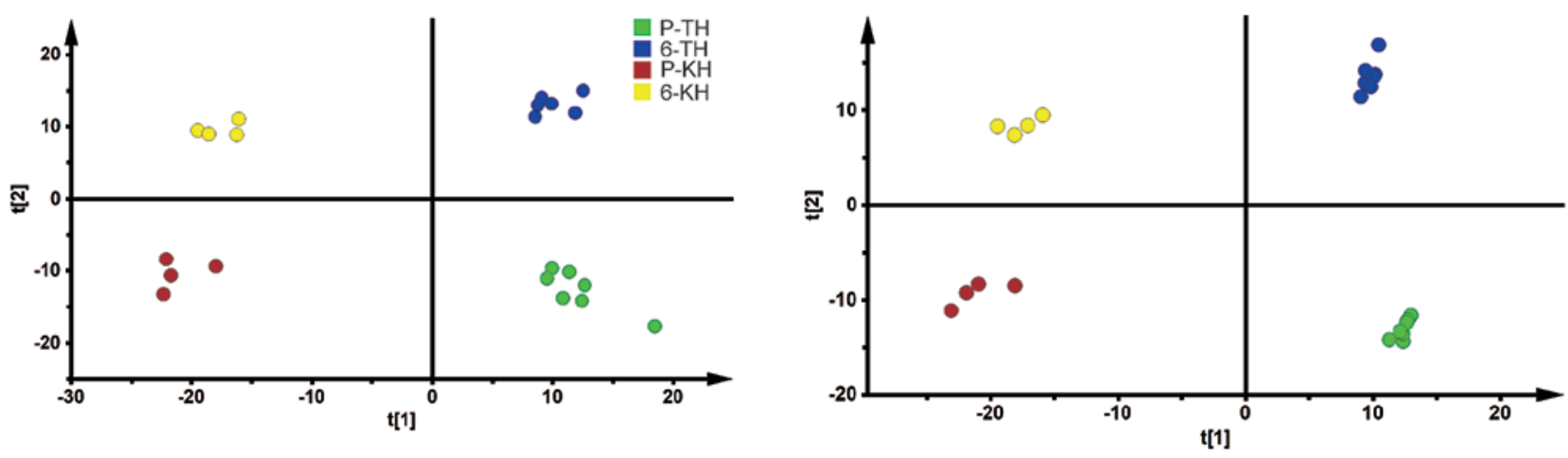

Figure 3. PCA and OPLSDA figures of participants suffering HAPC. (6: 6-month re-oxygenation; K, from location K; T, from point T; C, control groups; H, HAPC group). OPLS-DA, partial squares discriminant analysis; PCA, principal component analysis; high altitude polycythemia.

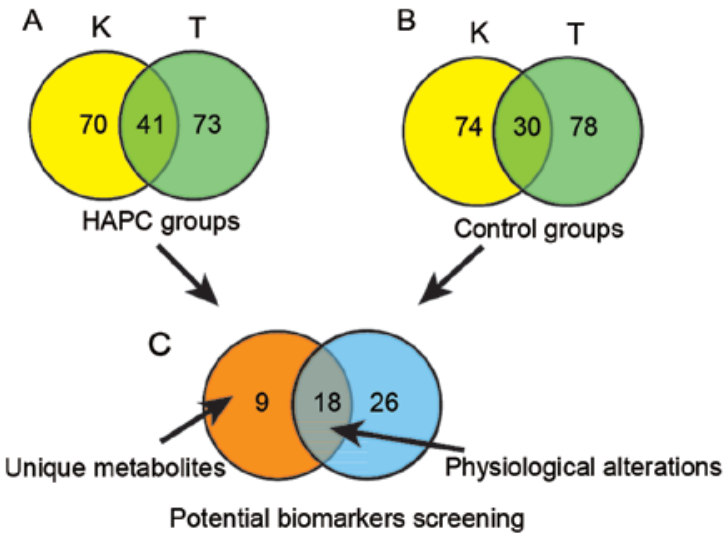

Figure 4. Differential metabolites identification by Venn figure. (A) showed distinguishing molecules with same variation tendency in HAPC groups in locations of $\mathrm{K}$ and $\mathrm{T}$. (B) represented the selection of significant metabolites from Control groups in locations of $\mathrm{K}$ and $\mathrm{T}$. (C) Screening potential biomarkers from previously selected distinguishing metabolites. HAPC, high altitude polycythemia.

Potential biomarkers screening. To further narrow down the impacts of environmental factors and raise the accuracy of our selection, a Venn diagram was executed to focus on the overlapping parts of differential molecules which were shared by two separating locations and possessed identical variation tendency. Metabolites shared by HAPC subjects from two separate locations were exhibited in Fig. 4A. Accordingly, identified in both control groups of two locations, the most perturbed molecules with identical alteration trends in physiological alterations were identified in Fig. 4B.

A significant goal of our study was to screen serum metabolites which contribute to significant $\mathrm{HB}$ reduction for HAPC subjects. Such biomarkers should meet the criteria of discriminating HAPC subjects before and after the intervention of re-oxygenation with none effects on normal physiological alterations. As shown in Fig. 4C, 9 metabolites in the red part of Fig. 4 varied uniquely in HAPC groups from both locations were identified. The detailed information of these biomarkers is listed in Table II. In addition, physiological changes during re-oxygenation were also showed Fig. 4C. The detailed information of these molecules is listed in Table III. The information of identifying these metabolites from the UPLC-QTOF/MS instrument is listed in Table IV.

Metabolic pathways analysis and affected networks identification. Metabolic pathway analysis was performed to reveal alterations of metabolites, which played a common role in 
Table III. The distinguishing metabolites enrolled in physiological changes of all subjects during re-oxygenation.

\begin{tabular}{lcccccccc}
\hline Molecules & P-value & Q-value & FC & VIP & P-value & Q-value & FC & VIP \\
\hline ESI+ & & & & & & & & \\
$\quad$ Indole & 0.0058 & 0.0029 & 0.6505 & 1.2301 & $5.04 \mathrm{E}-05$ & $3.53 \mathrm{E}-05$ & 0.5517 & 1.2358 \\
Succinic acid & 0.0003 & 0.0003 & 0.4928 & 1.4292 & $4.28 \mathrm{E}-07$ & $7.52 \mathrm{E}-07$ & 0.4487 & 1.4187 \\
Hypoxanthine & 0.0003 & 0.0003 & 0.4154 & 1.4173 & $1.07 \mathrm{E}-08$ & $4.29 \mathrm{E}-08$ & 0.4034 & 1.4678 \\
Phenylpyruvic acid & 0.0003 & 0.0003 & 0.4241 & 1.4370 & $1.85 \mathrm{E}-08$ & $6.60 \mathrm{E}-08$ & 0.3611 & 1.4684 \\
L-Phenylalanine & 0.0000 & 0.0000 & 7.3158 & 1.5196 & $1.37 \mathrm{E}-07$ & $3.01 \mathrm{E}-07$ & 8.4551 & 1.4653 \\
3-Phosphoglyceric acid & 0.0000 & 0.0000 & 3.5927 & 1.4906 & $6.90 \mathrm{E}-11$ & $1.15 \mathrm{E}-09$ & 3.0244 & 1.5277 \\
N-Acetyl-D-glucosamine & 0.0000 & 0.0000 & 0.0891 & 1.4888 & $4.65 \mathrm{E}-08$ & $1.23 \mathrm{E}-07$ & 0.1553 & 1.4811 \\
DHAP(18:0) & 0.0000 & 0.0001 & 0.2641 & 1.4747 & $3.44 \mathrm{E}-07$ & $6.50 \mathrm{E}-07$ & 0.2308 & 1.4586 \\
PA (20:4 (5Z,8Z,11Z,14Z)) & 0.0000 & 0.0001 & 3.8687 & 1.4721 & $1.86 \mathrm{E}-05$ & $1.57 \mathrm{E}-05$ & 2.7362 & 1.3258 \\
LysoPC (P-18:1 (9Z)) & 0.0019 & 0.0013 & 0.2447 & 1.2866 & $1.18 \mathrm{E}-05$ & $1.10 \mathrm{E}-05$ & 0.1747 & 1.3233 \\
LysoPC (P-18:0) & 0.0016 & 0.0011 & 0.3878 & 1.2779 & $5.53 \mathrm{E}-08$ & $1.41 \mathrm{E}-07$ & 0.2538 & 1.4688 \\
Tetrahydroaldosterone-3-glucuronide & 0.0022 & 0.0015 & 3.8443 & 1.2379 & $3.53 \mathrm{E}-06$ & $4.31 \mathrm{E}-06$ & 3.7355 & 1.3823 \\
Bilirubin & 0.0005 & 0.0005 & 0.2375 & 1.2987 & $2.76 \mathrm{E}-06$ & $3.55 \mathrm{E}-06$ & 0.1069 & 1.4108 \\
ESI- & & & & & & & & \\
3-Sulfinylpyruvic acid & 0.0000 & 0.0000 & 0.3117 & 1.4857 & $2.74 \mathrm{E}-05$ & $2.11 \mathrm{E}-05$ & 0.4880 & 1.3071 \\
Tryptamine & 0.0001 & 0.0001 & 0.1339 & 1.4537 & $2.38 \mathrm{E}-09$ & $1.73 \mathrm{E}-08$ & 0.5236 & 1.5287 \\
L-Dopa & 0.0006 & 0.0005 & 0.5062 & 1.3724 & $6.35 \mathrm{E}-05$ & $4.28 \mathrm{E}-05$ & 0.6258 & 1.2421 \\
2,3-Diacetoxypropyl stearate & 0.0000 & 0.0000 & 0.0684 & 1.4906 & $2.53 \mathrm{E}-06$ & $3.39 \mathrm{E}-06$ & 0.3996 & 1.3838 \\
Ganglioside GA1 (d18:1/9Z-18:1) & 0.0000 & 0.0001 & 0.3729 & 1.3886 & $1.81 \mathrm{E}-09$ & $1.46 \mathrm{E}-08$ & 0.2611 & 1.5264 \\
\hline
\end{tabular}

HAPC, high altitude polycythemia; FC, fold-change; VIP, variable importance project.

the hosts' response to re-oxygenation. The most disturbed biochemical pathways involved in normal physiological alterations after re-oxygenation is phenylalanine metabolism (Fig. 5A). In addition, metabolites set enrichment analysis on uniquely distinguishing molecules in the recovery phase of HAPC groups of two locations revealed that pentose phosphate pathway is the most perturbed pathways with $\mathrm{P}<0.05$ (Fig. 5B).

Validation of findings by comparing HAPC and Control groups. To explore if our findings also contributed to the progress of hypoxia-induced erythrocytosis, we comparatively studied expression patterns of crucial molecules in pentose phosphate pathway in HAPC and control groups in location $\mathrm{T}$ and K, separately. Further exaggerating variances of these metabolites, subjects with HB level lower than $20 \mathrm{~g} / \mathrm{dl}$ and those with HB larger than $21 \mathrm{~g} / \mathrm{dl}$ were enrolled in the comparison. As shown in Table V, molecules of 6-phosphogluconic acid, phosphoenolpyruvic acid, glucose 6-phosphate, phosphoribosyl pyrophosphate which are the essential participants of pentose phosphate pathway were elevated in control groups comparing to HAPC groups. All molecules showed significant alterations at least in one location. Small samples size may prevent part of differential metabolites from being apparent.

\section{Discussion}

Hypoxia-induced erythrocytosis is a severe complication caused by chronic hypoxemia, which adversely aggravates organ hypoxia and damage. Without any solid findings of pre-clinical researches to facilitate recovery of this syndrome, none pathophysiologic driven therapeutic strategies are available currently, except for the strategies of re-oxygenation. Therefore, we conducted metabolomics study based on HAPC model with the aim of unraveling mechanisms involved in the natural recovery phase of decreasing HB in plain environment, and searching for potential therapeutic biomarkers to alleviate erythrocytosis symptom.

Pentose phosphate pathway (PPP), acted as hexose monophosphate shunt, forms one of the main antioxidant cellular defense systems for individuals, and acts as a major provider of ribose phosphate to regulate cell redox rebalance and proliferative fate (15). As listed in Fig. 6, our results revealed upregulated PPP in recovery processes of HAPC.Increased PPP could reduce NADP to NADPH, which plays an important role in regulating glutathione (GSH) and further protects against reactive oxygen species (ROS), repairs oxidized proteins of essential participants in carrying oxygen in RBC, promotes re-balance between pro-oxidants and antioxidants $(16,17)$. Further, we validated our findings by comparing HAPC and control group directly and detected decreasing pentose phosphate pathway in HAPC subjects, which implied that PPP may be involved in the progression of hypoxia-induced erythrocytosis. D'Alessandro et al has demonstrated that hypoxia blocks metabolic diversion towards the PPP in RBC cells (18). However, hypoxia could result in mitochondria and endoplasmic reticulum dysfunction, over-activate NADPH oxidase, xanthine oxidase, and uncoupling nitric oxide synthase, which form oxidative stress responses (19-21). Thus, decreasing PPP 
A

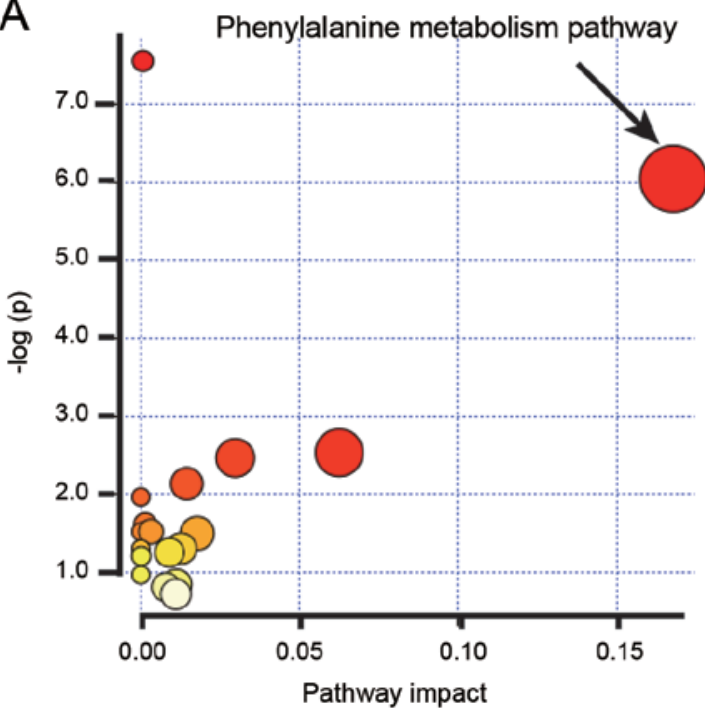

B

Metabolites sets enrichment

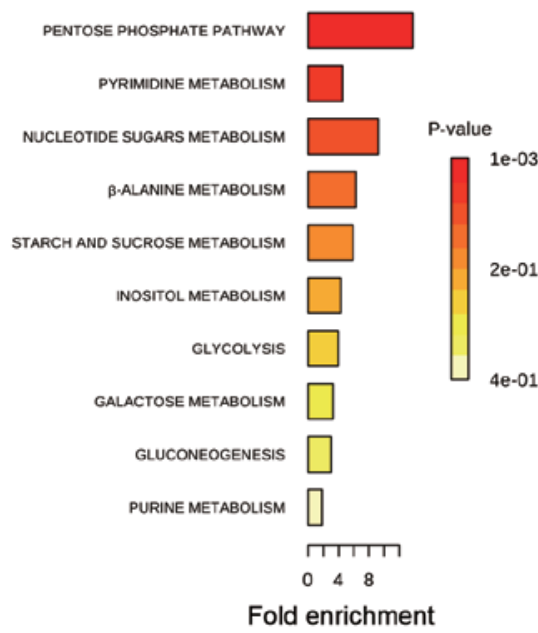

Figure 5. Summary of pathway analysis with MetaboAnalyst 3.0 (A) Altered metabolic pathway between of physiological recovery. (B) Enrichment analysis on uniquely distinguishing metabolites shared by HAPC subjects. HAPC, high altitude polycythemia.

A

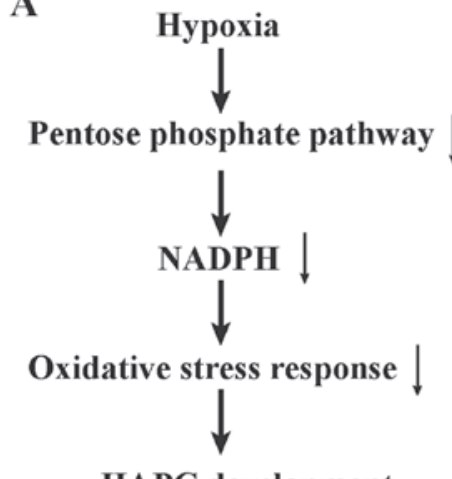

B

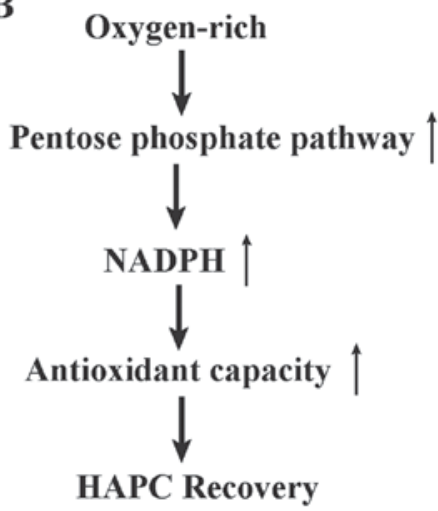

Figure 6. Schematic overview for functions of pentose phosphate pathway in the development and recovery phase of HAPC. (A) Downregulated pentose phosphate pathway could contribute to HAPC (B) Elevated pentose phosphate pathway are involved in the recovery of HAPC. HAPC, high altitude polycythemia.

impaired the capacities of RBC to cope with oxidative stress which lead to protein oxidation, lipid peroxidation, and DNA oxidation, further decreases ability in carrying oxygen in red blood cells and pathological hyperplasia of erythrocytes. In addition, decreasing PPP poses negative effects on GSH homeostasis, which constitutes another essential antioxidant system, to aggravate oxidative damage for cells (21). In summary, increasing PPP pathway may be a potential therapeutic target to halt the progression and promote the recovery of hypoxia-induced erythrocytosis.

In this study, we also identified disturbed phenylalanine metabolism in physiological changes during the process of re-oxygenation in all groups. Originated from exogenous supply via food, phenylalanine is an essential amino acid, which human body is unable to synthetize. The significantly elevated phenylalanine level observed in our enrolled subjects, was far from diagnostic standards of Phenylketonuria, and none symptoms of Phenylketonuria could be detected in these participants in our study. Tyrosine and phenylpyruvic acid are the major downstream metabolites of phenylalanine and maintain homeostasis in individuals (22). However, none identical alterations in serum tyrosine was observed in our study comparing to that of phenylalanine while phenylpyruvic acid was significantly impaired after the process of re-oxygenation (23). These paradoxical phenomena may due to increasing oxidative stress caused by re-oxygenation with elevated ROS and RNS, which further interfere the endogenous synthesis of antioxidants and influence metabolic enzyme activities (24). Previous reports have identified that the conversion from phenylalanine to tyrosine is catalyzed by the enzyme phenylalanine hydroxylase, which could be impaired by oxidative stress $(25,26)$. On the other hand, conversion from phenylalanine to phenylpyruvic acid exhibited a NAD $(\mathrm{H})$-dependent pattern, which was impaired by increasing oxidative stress and decreased significantly during re-oxygention $(27,28)$. Therefore, the metabolism of phenylalanine to phenylpyruvic acid and tyrosine may contribute to the accumulation of phenylalanine.

The elevated phenylalanine expression detected in our study posed both advantages and disadvantages during re-oxygenation process. On one hand, oxidative stress would be promoted with the rising phenylalanine expression by 
Table IV. The information of identifying these metabolites from the UPLC-QTOF/MS instrument.

\begin{tabular}{|c|c|c|c|}
\hline Number & Metabolite & $\operatorname{tr} / \mathrm{min}$ & $\mathrm{m} / \mathrm{z}$ \\
\hline \multicolumn{4}{|l|}{ ESI+ } \\
\hline 1 & Indole & 118.0650 & 5.88417 \\
\hline 2 & Succinic acid & 119.0730 & 5.885 \\
\hline 3 & Hypoxanthine & 137.0150 & 5.88483 \\
\hline 4 & Phenylpyruvic acid & 165.0100 & 5.885 \\
\hline 5 & Glucose 6-phosphate & 165.0100 & 0.733 \\
\hline 6 & L-Phenylalanine & 166.0270 & 0.920583 \\
\hline 7 & 3,4-Dihydroxyphenylglycol O-sulfate & 166.0270 & 0.684 \\
\hline 8 & 3-Phosphoglyceric acid & 187.0700 & 0.854583 \\
\hline 9 & LysoPE (0:0/22:2) & 187.0700 & 15.6952 \\
\hline 10 & L-Tryptophan & 205.0971 & 5.86033 \\
\hline 11 & N-Acetyl-D-glucosamine & 222.0290 & 5.8955 \\
\hline 12 & Phosphoribosyl pyrophosphate & 390.9591 & 0.608417 \\
\hline 13 & DHAP(18:0) & 437.1940 & 14.1175 \\
\hline 14 & PA (20:4 (5Z, 8Z, 11Z, 14Z)) & 487.3570 & 18.3125 \\
\hline 15 & LysoPC (P-18:1 (9Z)) & 506.3590 & 16.5833 \\
\hline 16 & LysoPC (P-18:0) & 508.3760 & 16.5149 \\
\hline 17 & Tetrahydroaldosterone-3-glucuronide & 541.3290 & 16.145 \\
\hline 18 & Bilirubin & 585.2710 & 11.2249 \\
\hline \multicolumn{4}{|l|}{ ESI- } \\
\hline 19 & 3-Sulfinylpyruvic acid & 149.9950 & 21.9133 \\
\hline 20 & Tryptamine & 159.1140 & 0.796111 \\
\hline 21 & L-Dopa & 196.0220 & 1.34443 \\
\hline 22 & All-trans-retinoic acid & 299.2016 & 20.4552 \\
\hline 23 & Uracil & 437.1940 & 1.29277 \\
\hline 24 & 2,3-Diacetoxypropyl stearate & 441.2370 & 14.0312 \\
\hline 25 & Deoxyribose 5-phosphate & 506.3590 & 9.88136 \\
\hline 26 & LysoPE (0:0/22:6) & 541.3290 & 16.1388 \\
\hline 27 & Ganglioside GA1 (d18:1/9Z-18:1) & 625.3080 & 15.3796 \\
\hline
\end{tabular}

$\mathrm{m} / \mathrm{z}$, mass-to-charge ratio.

Table V. The essential molecules in pentose phosphate pathway in HAPC and control groups at high altitude.

\begin{tabular}{lll}
\hline Metabolites & K_FC & T_FC \\
\hline 6-Phosphogluconic acid & 1.03 & $1.26^{\mathrm{c}}$ \\
Phosphoenolpyruvic acid & $1.34^{\mathrm{c}}$ & 1.182 \\
Glucose 6-phosphate & $1.41^{\mathrm{c}}$ & $1.57^{\mathrm{c}}$ \\
Phosphoribosyl pyrophosphate & $1.152^{\mathrm{c}}$ & 1.02
\end{tabular}

${ }^{a}$ The fold-change value calculated by Control/HAPC in $\mathrm{K}$ point; bthe fold-change value calculated by Control/HAPC in $\mathrm{T}$ point; ( ${ }^{c} \mathrm{P}$-value $<0.05$, P-value was calculated by student's t-test). HAPC, high altitude polycythemia.

increasing endogenous synthesis of reactive species, free radicals and interfering with the endogenous synthesis of enzymatic antioxidants (29). On the other hand, phenylalanine exhibits anti-hypertension and anti-cardiovascular remodeling by decreasing intracellular [Ca2+] and cardiovascular cells growth, which formed the basis for the unbeneficial structural reconstruction under prolonged hypoxic exposure (30). In addition, phenylalanine has been demonstrated to be involved in regulating metabolic conditions. Lin et al revealed that elevated phenylalanine was correlating to lower level of glucose in serum, with a significant insulinotropic effect, and contributed to the establishment of effective energy metabolic pathways (23). Increasing phenylalanine was also demonstrated to promote pulmonary function, with high correlation to FVC1 (31). Generally, as a physiologically regulated metabolite, appropriately higher expression of phenylalanine could alleviate the structural alterations and promote the recovery of metabolic patterns after the long-living in chronic hypoxia environment. The effects on oxidation-promoting may be reduced by enhancing the conversion from phenylalanine to meta-L-tyrosine (32). Phenylalanine warrants further studies in the process of hypoxia/re-oxygenation.

There are several limitations needed to be reconsidered in this study. Firstly, a relative small sample size may prevent 
parts of distinguishing metabolites from being apparent. Thus, instead of comparing subjects suffering hypoxia-induced erythrocytosis with control groups directly, we established a self-control experiment to improve efficiency and accuracy of our study. Secondly, the homogeneity of enrolled participants was not prerequisite for this study and heterogeneous population should be recruited, such as subjects in diverse sexes and age periods. Therefore, a larger metabolomics study combining validation experiments to verify our findings by comparing the HAPC with controls directly is now conducting by our group.

In conclusion, this is the first study in providing a comprehensive description of metabolic profiling in the recovery phase of hypoxia-induced polycythemia, and tracing along for half a year. Elevated pentose phosphate pathway may be involved in the recovery of HAPC, while downregulated pentose phosphate pathway could contribute to the progress of hypoxia-induced erythrocytosis. In addition, alterations in phenylalanine metabolism participate in the individuals' reaction to re-oxygenation.

\section{Acknowledgements}

The present study was supported by the Key Projects in the Military Science and Technology Pillar Program during the Thirteen 5-year Plan Period (AWS14C007), by National Natural Science Foundation of China (J1310001).

\section{References}

1. Chen Y, Jiang C, Luo Y, Liu F and Gao Y: Interaction of CARD14, SENP1 and VEGFA polymorphisms on susceptibility to high altitude polycythemia in the Han Chinese population at the Qinghai-Tibetan Plateau. Blood Cells Mol Dis 57: 13-22, 2016.

2. Tashi T, Feng T, Koul P, Amaru R, Hussey D, Lorenzo FR, RiLi G and Prchal JT: High altitude genetic adaptation in Tibetans: No role of increased hemoglobin-oxygen affinity. Blood Cells Mol Dis 53: 27-29, 2014.

3. Al-Sheikh M, Moradkhani K, Lopez M, Wajcman H and Préhu C: Disturbance in the HIF-1alpha pathway associated with erythrocytosis: Further evidences brought by frameshift and nonsense mutations in the prolyl hydroxylase domain protein 2 (PHD2) gene. Blood Cells Mol Dis 40: 160-165, 2008.

4. Frey H, Moreth K, Hsieh LT, Zeng-Brouwers J, Rathkolb B Fuchs H, Gailus-Durner V, Iozzo RV, de Angelis MH and Schaefer L: A novel biological function of soluble biglycan: Induction of erythropoietin production and polycythemia. Glycoconj J 34: 393-404, 2017.

5. Azad P, Zhao HW, Cabrales PJ, Ronen R, Zhou D, Poulsen O, Appenzeller O, Hsiao YH, Bafna V and Haddad GG: Senp1 drives hypoxia-induced polycythemia via GATA1 and Bcl-xL in subjects with Monge's disease. J Exp Med 213: 2729-2744, 2016.

6. Jiang C, Cui J, Liu F, Gao L, Luo Y, Li P, Guan L and Gao Y: Mitochondrial DNA $10609 \mathrm{~T}$ promotes hypoxia-induced increase of intracellular ROS and is a risk factor of high altitude polycythemia. PLoS One 9: e87775, 2014.

7. Köhler D and Dellweg D: Polycythemia. Dtsch Med Wochenschr 135: 2300-2303, 2010 (In German).

8. Tian H, Lam SM and Shui G: Metabolomics, a powerful tool for agricultural research. Int J Mol Sci 17: pii: E1871, 2016

9. Lima AR, Bastos Mde L, Carvalho M and Guedes de Pinho P: Biomarker discovery in human prostate cancer: An update in metabolomics studies. Transl Oncol 9: 357-370, 2016.

10. McClain DA, Abuelgasim KA, Nouraie M, Salomon-Andonie J, Niu X, Miasnikova G, Polyakova LA, Sergueeva A, Okhotin DJ, Cherqaoui $\mathrm{R}$, et al: Decreased serum glucose and glycosylated hemoglobin levels in patients with Chuvash polycythemia: A role for HIF in glucose metabolism. J Mol Med (Berl) 91: 59-67, 2013.
11. Liao WT, Liu B, Chen J, Cui JH, Gao YX, Liu FY, Xu G, Sun BD, Zhang EL, Yuan ZB, et al: Metabolite modulation in human plasma in the early phase of acclimatization to hypobaric hypoxia. Sci Rep 6: 22589, 2016.

12. Smith CA, O'Maille G, Want EJ, Qin C, Trauger SA, Brandon TR, Custodio DE, Abagyan R and Siuzdak G: METLIN: A metabolite mass spectral database. Ther Drug Monit 27: 747-751, 2005.

13. Wishart DS, Jewison T, Guo AC, Wilson M, Knox C, Liu Y, Djoumbou Y, Mandal R, Aziat F, Dong E, et al: HMDB 3.0-the human metabolome database in 2013. Nucleic Acids Res 41 (Database Issue): D801-D807, 2013.

14. Xia J, Sinelnikov IV, Han B and Wishart DS: MetaboAnalyst 3.0-making metabolomics more meaningful. Nucleic Acids Res 43 (W1): W251-W257, 2015.

15. Riganti C, Gazzano E, Polimeni M, Aldieri E and Ghigo D: The pentose phosphate pathway: An antioxidant defense and a crossroad in tumor cell fate. Free Radic Biol Med 53: 421-436, 2012.

16. van Zwieten R, Verhoeven AJ and Roos D: Inborn defects in the antioxidant systems of human red blood cells. Free Radic Biol Med 67: 377-386, 2014.

17. Karasawa T, Saito T, Ueno $Y$, Sugimoto $M$ and Soga $T$ : Metabolome analysis of erythrocytes from patients with chronic hepatitis $C$ reveals the etiology of ribavirin-induced hemolysis. Int J Med Sci 10: 1575-1577, 2013

18. D'Alessandro A, Gevi F and Zolla L: Red blood cell metabolism under prolonged anaerobic storage. Mol Biosyst 9: 1196-1209, 2013.

19. Zhou L, Chen P, Peng Y and Ouyang R: Role of oxidative stress in the neurocognitive dysfunction of obstructive sleep apnea syndrome. Oxid Med Cell Longev 2016: 9626831, 2016.

20. Kim M, Han CH and Lee MY: NADPH oxidase and the cardiovascular toxicity associated with smoking. Toxicol Res 30: 149-157, 2014.

21. Rauchová H, Vokurková M and Koudelová J: Hypoxia-induced lipid peroxidation in the brain during postnatal ontogenesis. Physiol Res 61 (Suppl 1): S89-S101, 2012.

22. Gostner JM, Becker K, Kurz K and Fuchs D: Disturbed amino acid metabolism in HIV: Association with neuropsychiatric symptoms. Front Psychiatry 6: 97, 2015.

23. Lin X, Zhao L, Tang S, Zhou Q, Lin Q, Li X, Zheng H and Gao H: Metabolic effects of basic fibroblast growth factor in streptozotocin-induced diabetic rats: A 1H NMR-based metabolomics investigation. Sci Rep 6: 36474, 2016.

24. Kim YW and Byzova TV: Oxidative stress in angiogenesis and vascular disease. Blood 123: 625-631, 2014

25. Stevens JP, Churchill T, Fokkelman K, Haase E, Idikio H, Korbutt G, Bigam DL and Cheung PY: Oxidative stress and matrix metalloproteinase-9 activity in the liver after hypoxia and reoxygenation with $21 \%$ or $100 \%$ oxygen in newborn piglets. Eur J Pharmacol 580: 385-393, 2008

26. Fuchs JE, Huber RG, von Grafenstein S, Wallnoefer HG, Spitzer GM, Fuchs D and Liedl KR: Dynamic regulation of phenylalanine hydroxylase by simulated redox manipulation. PLoS One 7: e53005, 2012.

27. Jiang W and Fang BS: Construction and evaluation of a novel bifunctional phenylalanine-formate dehydrogenase fusion protein for bienzyme system with cofactor regeneration. J Ind Microbiol Biotechnol 43: 577-584, 2016.

28. Vilaseca MA, Farré C and Ramón F: Phenylalanine determined in plasma with use of phenylalanine dehydrogenase and a centrifugal analyzer. Clin Chem 39: 129-131, 1993.

29. Rocha JC and Martins MJ: Oxidative stress in phenylketonuria: Future directions. J Inherit Metab Dis 35: 381-398, 2012.

30. Zhao G, Li Z and Gu T: Antihypertension and anti-cardiovascular remodeling by phenylalanine in spontaneously hypertensive rats: Effectiveness and mechanisms. Chin Med J (Engl) 114: 270-274, 2001.

31. Førli L, Pedersen JI, Bjørtuft, Vatn M, Kofstad J and Boe J: Serum amino acids in relation to nutritional status, lung function and energy intake in patients with advanced pulmonary disease. Respir Med 94: 868-874, 2000.

32. Molnár GA, Kun S, Sélley E, Kertész M, Szélig L, Csontos C, Böddi K, Bogár L, Miseta A and Wittmann I: Role of tyrosine isomers in acute and chronic diseases leading to oxidative stress-A Review. Curr Med Chem 23: 667-685, 2016. 